\title{
Comparative proteomics reveals different protein expression in platelets in patients with alcoholic liver cirrhosis
}

Nima Haji Begli

University Hospital Heidelberg

Cora Freund

University Hospital Heidelberg

Karl-Heinz Weiss

Salem Medical Center

Daniel Gotthardt

University Hospital Heidelberg

Andreas Wannhoff ( $\square$ Andreas.Wannhoff@rkh-kliniken.de)

Hospital Ludwigsburg

\section{Research Article}

Keywords: Platelet Proteomics, Alcoholic Liver Cirrhosis, Rab-7a, RANBP1, RhoGDI1, 14-3-3 gamma

Posted Date: November 17th, 2022

DOI: https://doi.org/10.21203/rs.3.rs-1344377/v2

License: (9) This work is licensed under a Creative Commons Attribution 4.0 International License. Read Full License 


\section{Abstract \\ Background}

The role of platelets in disease progression as well as the function of platelets as part of the haemostatic and immunological system in patients with liver cirrhosis is only incompletely understood. This is partly due to difficulties in assessing platelet function. Proteome analyses of platelets have been used to further investigate the role of platelets in other diseases.

\section{Aim}

To assess possible changes in the platelet proteome during different stages of alcohol induced liver cirrhosis compared to healthy donors.

\section{Patients and Methods}

A $45 \mathrm{ml}$ blood sample was drawn from 18 participants aged 18-80 years evenly divided into three groups of healthy donors, patients with less advanced alcohol induced liver cirrhosis (Child-Pugh $<7$ ) and patients with advanced liver cirrhosis (Child-Pugh > 10). The blood was processed to isolate platelets and perform subsequent two-dimensional gel-electrophoresis using a SYPRO ${ }^{\text {TM }}$ Ruby dye. After computational analysation significantly in- or decreased protein spots (defined as a two-fold abundance change between different study cohorts and ANOVA $<0.05$ ) were identified via liquid chromatography-mass spectrometry (LCMS) and searching against human protein databases.

\section{Results}

The comparative analysis identified four platelet proteins with progressively decreased protein expression in patients with liver cirrhosis. More specifically Ras-related protein Rab-7a (Rab-7a), Ran-specific binding protein 1 (RANBP1), Rho GDP-dissociation inhibitor 1 (RhoGDI1), and 14-3-3 gamma.

\section{Conclusion}

There is significant change in protein expression in the platelet proteome throughout the disease progression of alcohol induced liver cirrhosis. The identified proteins are possibly involved in haemostatic and immunoregulatory function of platelets.

\section{Introduction}


Chronic liver disease and especially liver cirrhosis is usually accompanied by multiple changes within the haemostatic system. These include abnormalities of multiple coagulation factors affecting pro- and anticoagulant factors as well as the fibrinolytic system, thrombocytopenia and potentially thrombocytopathy $(1,2)$. Conventional coagulation tests typically show an increased international normalized ratio (INR) and a reduced platelet count and thus patients with liver cirrhosis were long believed to have an acquired coagulation disorder with increased risk of bleeding and were thought to be naturally anticoagulated (3). It is nowadays no longer believed that patients with liver cirrhosis are "naturally" anticoagulated but rather have a re-balanced haemostatic system that might lead to bleeding or thrombosis through changes in pro- and anticoagulant factors (4) and therefore go from a healthy balanced state to a re-balanced state throughout their disease progression. There also is evidence that suggests increased coagulation in patients with cirrhosis and these patients do show an increased risk of peripheral thrombosis (5). While the coagulation system is well studied in liver cirrhosis and thrombocytopenia is known to be a hallmark of end-stage liver disease (ESLD), less is known about platelet function and the presence of thrombocytopathy (6).

Furthermore, research in recent years has increasingly shown that platelets not only play a primary role in haemostasis as described above, but also participate in the immune response, the resolution of inflammation $(7,8)$ and angiogenesis $(9)$. In addition, recent data shows that in liver fibrosis, platelets are involved in the development of hepatic fibrosis via complex interactions with hepatic stellate cells (HSC) $(10,11)$. Interestingly both activating and inhibiting HSC are consequently leading to hepatic fibrosis through a continuous injury response.

Thus, it is of high interest to shed a light on possible changes in platelet function in patients with liver cirrhosis; yet measurement of platelet function and activity remains challenging. Investigation of the platelet proteome by means of Proteomics in various diseases has been shown to be possible and promising $(12,13)$.

We hypothesized that there is presence of thrombocytopathy in patients with ESLD and aimed to investigate this by performing analyses of the platelet proteome in patients with alcohol induced liver cirrhosis and healthy controls.

\section{Materials And Methods}

\section{Study protocol}

Platelet analysis was planned in patients with alcoholic liver cirrhosis as well as in a control cohort (Group Contr) of participants without liver disease. The group of patients with cirrhosis was planned to be split in two subgroups with less advanced disease (Group Cirr1; defined as Child Pugh score $\leq 7$ ) and with advanced disease (Group Cirr2; Child Pugh score > 10). A total of 18 participants, six in each of the three groups, were planned to be included. Exclusion criteria were as followed: (1) platelet count $<50.000$, (2) diagnosis of thrombotic diseases, (3) use of antithrombotic medication in the last 4 weeks or 
scheduled to start of such medication and/or history of anticoagulation, (4) malignant disease - patients were as well excluded per protocol during a 12 month follow up period if there was a new cancer diagnosis, and (5) relapse in alcohol consumption in the last four months prior to enrolment - in order to exclude acute myelosuppressive changes of alcohol consumption. Patients treated at Department of Internal Medicine IV of University Hospital Heidelberg were screened for inclusion. Six healthy donors were paired by age and sex and were enrolled in the study as controls.

The study was previously approved by the ethics committee of the University of Heidelberg and all participants provided written informed consent prior to inclusion in the study.

\section{Plasma collection, platelet separation and storage}

In total five vials each containing $9 \mathrm{ml}$ blood sample and $1 \mathrm{ml}$ sodium citrate stock solution (equalling a final concentration of $10 \%(\mathrm{v} / \mathrm{v})$ with anticoagulant) were drawn as usual for the control and patient group. Each blood sample was processed individually. $1 \mathrm{ml}$ citrate dextrose solution (ACD) stock solution was added to each vial prior to centrifugation at $200 \mathrm{xg}$ (times gravity) for $20 \mathrm{~min}$. The upper third of the platelet rich plasma (PRP) was transferred to a new tube to avoid contamination with leukocytes and Prostacyclin $(2.5 \mathrm{mM})$ was added to avoid platelet activation. The sample was centrifuged at $1000 \mathrm{xg}$ for $10 \mathrm{~min}$. The resulting pellet was washed with modified Tyrodes-HEPES-buffer ((134 mM NaCl, $0.34 \mathrm{mM}$ $\mathrm{Na}_{2} \mathrm{HPO}_{4}, 2.9 \mathrm{mM} \mathrm{KCl}, 12 \mathrm{mM} \mathrm{NaHCO}_{3}, 20 \mathrm{mM}$ HEPES, $5 \mathrm{mM}$ glucose, $1 \mathrm{mM} \mathrm{MgCl}$, 1 mM EGTA, $10 \mathrm{M}$ indomethacin, $\operatorname{ACD}(7 \%, \mathrm{v} / \mathrm{v}), \mathrm{pH} 7.3)$ and again centrifuged at $1000 \mathrm{~g}$ for 10 minutes. Then, platelets were resuspended at a concentration of $2 \times 10^{8}$ cells $/ \mathrm{ml}$ with Tyrodes-HEPES buffer, incubated for 30 minutes at room temperature, and centrifuged at $10000 x g$ for 2 minutes. Finally, protease and phosphatase inhibitor (Halt ${ }^{\text {tM }}$ Protease and Phosphatase Inhibitor Cocktail, Thermo Fisher Scientific, Waltham, USA) were added and resulting pellets were stored at $-80^{\circ} \mathrm{C}$ for further analysis.

\section{Cell lysis}

Prior to further analysis each sample was thawed on iced. The pellets were then suspended in $100 \mu \mathrm{l}$ $0.9 \% \mathrm{NaCl}$ and spun for 2 minutes. Following, each pellet was added to $50 \mu \mathrm{lTCA}$ and resuspended with $350 \mathrm{ml}$ lysis buffer ((9.5 M Urea, 4\% CHAPS, $65 \mathrm{mM}$ DTT, $0.2 \%$ carrier ampholyte, and $20 \mu \mathrm{l} / \mathrm{ml}$ protease inhibitor cocktail).

\section{Isoelectric focusing}

The first step of isoelectric focusing (IEF) was done using immobilized pH gradient (IPG) strips (nonlinear gradient, $18 \mathrm{~cm}, \mathrm{pH}$ 3-10, Thermo Fisher Scientific, Waltham, USA). Each strip was rehydrated for $5 \mathrm{~h}$ at $30 \mathrm{~V}, 6 \mathrm{~h}$ at $60 \mathrm{~V}$ and $2 \mathrm{~h}$ at $100 \mathrm{~V}$. IEF was conducted at $200 \mathrm{~V}, 500 \mathrm{~V}$ and $1000 \mathrm{~V}$ for each $1,5 \mathrm{~h}$ followed by a gradient from $1000 \mathrm{~V}$ to $8000 \mathrm{~V}$ and focusing at $8000 \mathrm{~V}$ for $8 \mathrm{~h}$ to reach a total of approx. $60-80 \mathrm{kV}$. After completion of IEF, the IPG strips were immediately used for further analysis.

\section{2-DE Analysis}


Equilibration was performed via incubation of the strips in equilibration buffer for 20 minutes with gentle shaking (1\% w/v DTT, 50 mM, pH 6.8 Tris-HCl, 6 M Urea, 30\% Glycerol, 2\% SDS, bromophenol blue) followed by a second 20 min equilibration in ( $2.5 \% \mathrm{w} / \mathrm{v}$ IAA instead of DTT). The strips were then added for separation in the second dimension (molecular weight) to $12.5 \%$ acrylamide gels. Bromphenol blue was added to visualize the progress of the separation process. PageRuler Plus Prestain Protein (10 to 250 $\mathrm{kDa}$ ) was used as a protein marker. The separation was done as soon as the blue front reached the bottom. Following two fixation steps of each 30 minutes by placing the gels in a $50 \%$ methanol and $7 \%$ acetic acid solution visualization was done by SYPRO ${ }^{\text {TM }}$ Ruby (Thermo Fisher Scientific, Waltham, USA) protein gel stain following the staining method recommended by the manufacturer. Each sample was run in both a technical and biological duplicate to ensure reproducibility.

\section{Image acquisition and data analysis}

The image analysis was conducted with Image Master 2 (General Electric, Boston, USA). The resolution was 530 pixels per millimetre. Several parameters were fixed to ensure comparable data for quantitative analysis. Each subgroup (controls, mild and advanced liver cirrhosis) was grouped creating a reference gel for further analysis following the instructions of the developer. Grouping was done according to the proprietary parameters pre-set in the program. Internal software filters excluded experimental artifacts. Furthermore, each gel was resized manually to ensure best possible comparability. Spots with a 2 -fold abundance change and an ANOVA $<0.05$ were accepted as statistically significant and selected for protein identification. Increased volume by $50 \%$ was defined as up-regulation and decreased volume by $50 \%$ was defined as down-regulation.

\section{In-Gel-Digestion and Peptidextraction}

Protein digestion and LC-MS measurement were done as previously explained elsewhere (14). In short, protein bands of interest were excised manually from the reference gels. Following a reduction with 40 $\mathrm{mM}$ dithiothreitol and alkylation with $50 \mathrm{mM}$ iodoacetamide gel pieces were dehydrated. Trypsin solution (sequencing grade, Thermo-Fisher, Rockford, USA) was added to the dry gel pieces and incubated over night at 37 degrees Celsius. The reaction was quenched by addition of $20 \mu \mathrm{L}$ of $0.1 \%$ trifluoroacetic acid (TFA; Biosolve, Valkenswaard, Netherlands). The supernatant was dried in a vacuum concentrator before LC-MS analysis.

\section{LC-MS measurements}

Nanoflow LC-MS analysis was performed with NanoAcquity UPLC liquid chromatography (Waters, Eschborn, Germany) system coupled to an Orbitrap XL (Thermo-Fischer, Bremen, Germany). Samples were loaded to a NanoAcquity Symmetry C18 Trap column (particle size $5 \mu \mathrm{m}$, inner diameter $180 \mu \mathrm{m} \mathrm{x}$ $20 \mathrm{~mm}$, Waters) at a flow of $15 \mu \mathrm{L} / \mathrm{min}$ for $5 \mathrm{~min}$ of $0.5 \%$ solvent B. Peptides were separated using a NanoAcquity M-Class peptide BEH C18 analytical column (particle size $1.7 \mu \mathrm{m}$, inner diameter $75 \mu \mathrm{m} \mathrm{x}$ $250 \mathrm{~mm}$, Waters) with a $1 \mathrm{~h}$ linear gradient $(3-40 \% \mathrm{~B}$ ) with a flow rate of $300 \mathrm{~nL} / \mathrm{min}$. Solvent A was $0.1 \%$ formic acid (FA; ProteoChem, Denver, CO, USA) in H2O (Biosolve) and solvent B was composed of $0.1 \%$ 
FA (ProteoChem), 10\% H2O (Biosolve) and 89.9\% ACN (Biosolve). The mass spectrometer was operated in data-dependent acquisition mode, automatically switching between MS and MS 2. MS spectra $(\mathrm{m} / \mathrm{z}$ $400-1600)$ were acquired in the Orbitrap at $60,000(\mathrm{~m} / \mathrm{z} 400)$ resolution, with an automatic gain control (AGC) target value of $5 \times 10$ and maximal ion injection time (IT) $50 \mathrm{~ms}$. Collision induced dissociation MS 2 spectra were generated for up to 10 precursors with normalized collision energy of $35 \%$ in the ion trap. The MS 2 AGC target value was set to 104 with a maximum IT of $100 \mathrm{~ms}$.

\section{Protein identification}

Raw files were analyzed using Proteome Discoverer with the Sequest (Thermo Fisher Scientific, San Jose, USA; version 2.2). Sequest was set up to search against Uniprot human databases (June 2017) with trypsin as the digestion enzyme. A fragment ion mass tolerance was set to $0.50 \mathrm{Da}$ and a parent ion mass tolerance to $10 \mathrm{ppm}$. Carbamidomethyl of cysteine was specified as a fixed modification. Deamidation of asparagine and glutamine, oxidation of methionine and acetyl of the N-terminus were specified as variable modifications. The peptide and protein identity were verified by Scaffold (version Scaffold_4.8.4, Proteome Software Inc., Portland, USA). Peptide identifications were accepted if they could be established at greater than $95.0 \%$ probability by the Peptide Prophet algorithm (15) with Scaffold delta-mass correction. Protein identifications were accepted if they could be established at greater than $95.0 \%$ probability and contained at least 2 identified peptides. Protein probabilities were assigned by the Protein Prophet algorithm (16). Proteins that contained similar peptides and could not be differentiated based on MS/MS analysis alone were grouped to satisfy the principles of parsimony.

\section{Statistical analysis}

All data are expressed as mean with standard deviation (SD). Statistical analysis was conducted by SPSS Version 22 (IBM Corp., Armonk, USA) as well as Image Master 2 (General Electric, Boston, USA). A p-value less than 0.05 was defined as statistically significant.

\section{Results}

\section{Study cohort}

Between March 2015 and February 2017, 18 participants were included, six in each subgroup. Participants had a mean age of 52 (range: 39-60) and 9 participants (50.0\%) were male. Baseline characteristics of all three subgroups are presented in Table 1. 
Table 1

Study cohort characteristics

\begin{tabular}{|llll|}
\hline & Control & Less advanced & Advanced \\
\hline Sex $(\mathrm{m} / \mathrm{f})$ & $3 / 3$ & $3 / 3$ & $3 / 3$ \\
\hline $\begin{array}{l}\text { Age } \\
\text { (Range/Mean) }\end{array}$ & $40-60 / 50 \mathrm{y}$ & $39-60 / 51.5 \mathrm{y}$ & $48-59 / 54.5 \mathrm{y}$ \\
\hline $\begin{array}{l}\text { Platelet Count/nl } \\
\text { (Range/Mean) }\end{array}$ & $193-352 / 262$ & $142-297 / 207$ & $93-201 / 134$ \\
\hline $\begin{array}{l}\text { Red Blood Cell Count/pl } \\
\text { (Range/Mean) }\end{array}$ & $4.4-6.1 / 5.3$ & $4.2-6 / 5.1$ & $3.9-5.2 / 4.7$ \\
\hline $\begin{array}{l}\text { White Blood Cell Count/nl } \\
\text { (Range/Mean) }\end{array}$ & $4.3-10.3 / 8.3$ & $3.9-12.2 / 8.2$ & $3.1-11.4 / .7 .7$ \\
\hline
\end{tabular}

Table 1 Table containing the characteristics of the study cohort. Red blood cell count and white blood cell count rounded to first decimal.

\section{2-DE profiles of healthy volunteers and mild/moderate liver cirrhosis patients}

The group of healthy patients was named Contr and the liver cirrhosis patients were named Cirr1 and Cirr2 respectively. Highly consistent and reproducible gel images were obtained for platelet samples of all three groups (Contr, Cirr1, and Cirr2), as shown in Fig. 1. For the platelet samples of Contr an average of 1577 spots were resolved. For the platelet samples of liver cirrhosis averages of 1752 (Cirr1) and 1355 (Cirr2) spots were resolved. In total 220 spots matched between all the groups.

\section{Differential platelet 2-DE profiles between healthy volunteers and cirrhosis patients}

In total nine protein spots with varying degrees of increases of protein expression were shown to be statistically significant $(p<0.01)$. These were spots $10,11,29,143,153,306,311,312$ and 315.

\section{Identification of the various 2-DE spots by mass spectrometry}

These expressed and statistically significant proteins were manually excised, subjected to in-gel digestion and analyzed by MALDI-TOF-MS as described earlier. Spots 10,11, 143, 153 and 306 were mixed spots. Different proteins were detectable by mass spectrometry in the analysis and thus a more precise differentiation was not possible. The remaining spots successfully showed platelet proteins which were identified as followed: Spot 29 (ras-related proteins eg. Rab 7a), spot 311 (Ran-specific GTPase-activating 
protein), spot 312 (Rho GDP-dissociation inhibitor 1) and spot 315 (14-3-3 gamma). All the aforementioned spots were down-regulated (2.7-10 fold decrease, $p$-value ranging from 0.001 to 0.4 ). The special characteristics of aforementioned spots are described in Table 2.

Table 2

List of proteins of significance

\begin{tabular}{|llllll|}
\hline Spot & Name & $\begin{array}{l}\text { MW } \\
\text { gel } \mathbf{1}^{\mathbf{t} \text { theory }} \mathbf{2}\end{array}$ & $\begin{array}{l}\text { p- } \\
\text { value }\end{array}$ & Fold & Function \\
\hline 29 & Ras-related prot. & $20 / 20-24$ & 0.01 & 7,3 & $\begin{array}{l}\text { Rab protein signal } \\
\text { transduction }\end{array}$ \\
\hline 311 & $\begin{array}{l}\text { Ran-specific GTPase- } \\
\text { activating protein }\end{array}$ & $\begin{array}{l}\text { Approx } \\
22 / 23\end{array}$ & 0.001 & 3 & $\begin{array}{l}\text { nucleocytoplasmic } \\
\text { transport }\end{array}$ \\
\hline 312 & $\begin{array}{l}\text { Rho GDP-dissociation } \\
\text { inhibitor 1 }\end{array}$ & $\begin{array}{l}\text { Approx } \\
23 / 23\end{array}$ & 0.01 & 10 & GDP/GTP exchange \\
\hline 315 & 14-3-3 gamma & $\begin{array}{l}\text { Approx } \\
27 / 29\end{array}$ & 0.04 & 2,7 & $\begin{array}{l}\text { Adapter protein in } \\
\text { signaling pathways }\end{array}$ \\
\hline
\end{tabular}

Table 2 List of proteins accepted as statistically significant showing an at least 2-fold abundance change and a p-value of $<0.05{ }^{1}$ Molecular weight found in gel ${ }^{2}$ Molecular weight found in theory

In total four proteins with significantly decreased protein expression were identified in the analysis between patients with liver cirrhosis and healthy donors. These down-regulated proteins were Ras-related protein 7a (Rab-7a; Protein Accession Number: P51149), Ran-specific GTPase activating protein (RANBP1; Protein Accession Number: P43487), Rho-GDP dissociation inhibitor 1 (rho GDI 1; Protein Accession Number: P52565) and 14-3-3 gamma (Protein Accession Number: P61981). Among these proteins Rab-7a belongs to the Ras-related protein family which influences the RAB protein signal transduction, RANBP1 plays a role in RAN-dependent nucleocytoplasmic transport, rho GDI 1 controls Rho proteins homeostasis and furthermore regulates the GDP/GTP exchange reaction of the Rho proteins. Finally, 14-3-3 gamma belongs to the 14-3-3 protein family implicated in the regulation of various general and specialized signalling pathways. To our knowledge all the aforementioned proteins were previously found in platelet proteomics studies.

\section{Discussion}

In this study we conducted a comprehensive platelet proteomic analysis from patients with compensated and decompensated alcohol induced liver cirrhosis and non-cirrhotic controls.

The comparative analysis identified four platelet proteins, namely Ras-related protein Rab-7a (Rab-7a), Ran-specific binding protein 1 (RANBP1), Rho GDP-dissociation inhibitor 1 (RhoGDI1), and 14-3-3 gamma with progressively decreased protein expression in patients with liver cirrhosis. This indicates that alcoholic liver cirrhosis is associated with changes in protein expression in platelets These proteins are 
either directly and or indirectly linked to pathways associated with the haemostatic and possibly immunoregulatory function of platelets. As a consequence there might be altered haemostatic function of platelets in cirrhosis(i.e., thrombocytopathy) and a change in immunoregulatory function of platelets might play a role in cirrhosis progression.

Rab-7a is part of the most abundant family of small GTPases. Originally described as Ras-like proteins in the brain (Rab) these GTPases are often referred to as switches and regulators of vesicle trafficking in both endocytic and exocytic pathways. Rab-7a specifically plays a role in advanced stages of endosomal vesicle trafficking in platelets and is associated with advanced endocytic compartments and transport to lysosomes (17). Consequently, GTPases are an important component of platelet function in both haemostasis and immunological regulation (18). Although the precise process of Rab7a in platelet function and membrane trafficking remains unclear. Furthermore, the pathways of interaction and influence of Rab7a in regard to the platelet granule secretion warrant further research. But the consequently influenced process of platelet granule secretion and the composition of its granules play a role in haemostatic and possible immunological pathways (19).

The function of RANBP1 in platelets is currently unclear but the protein itself was identified in platelets in the past (20). It is known that RANBP1 is a Ran/TC4-binding protein and interacts especially with GTPcharged Ras-like nuclear (RAN) (21). RAN is the most abundant intracellular small GTPase. RAN acts as a molecular switch that alternates between an active GTP-bound and an inactive GDP-bound state. As already mentioned with Rab-7a the specific pathways of regulation are unclear.

The third protein with progressively decreased protein expression Rho GDP-dissociation inhibitor 1 is one of two Rho GDI dissociation proteins (RhoGDIs) expressed in platelets (22). These RhoGDIs are responsible for tuning and adjusting Rho GTPase activity (23). Due to its high expression (approximately 21000 copies per platelet) Rho GDP-dissociation inhibitor 1 provides a plausible candidate for the inhibitory regulation of Rho GTPases $(22,24)$. These GTPases are part of the Rho family and are small signalling $\mathrm{G}$ proteins and a subfamily of the Ras (Rat sarcoma virus) superfamily. In the last decades members of the RhoGTPase family were associated with changes in platelet physiology, the regulation of granule secretion as well as aggregation. Rho GTPases are known key players in cytoskeletal dynamics and therefore serve a critical role in platelets physiology and subsequently vital interactions like haemostasis and inflammation via modulation of platelet activation programs (24). A down regulation of RhoGDI1 is also seen in patients with hepatocellular carcinoma and is used as a prognostic factor (25). Through its inhibitory regulation Rho GDI-dissociation inhibitor 1 likely affects the various roles of platelets.

Lastly 14-3-3 gamma is part of the 14-3-3 family of proteins which play a substantial role in signal transduction pathways of eukaryotic cells. Members of the 14-3-3 protein family were previously described in platelets (26). They play a variety of regulatory roles in various phosphorylation-dependent signalling pathways, among others G-protein signalling (27). More specifically 14-3-3 proteins interact with several phosphoserine-dependent binding sites in glycoprotein Ib-IX complex (GPIb-IX), the major 
platelet adhesion receptor, thus affecting and possibly indirectly regulating its interplay with von Willebrand factor (VWF) and mediating platelet activation (28). One of these interplays is the platelet activation-dependent fibrin formation which can be relevant in haemostasis (29). Although 14-3-3 zeta is the main part of the 14-3-3 protein family interacting, all six 14-3-3 isotypes are able to bind to GPIb-IX (30). The interaction of members of the 14-3-3 protein family with GPIb-IX furthermore plays a critical role in enabling the platelet response to low concentrations of thrombin (31).

Thrombocytopenia is one of the most common haematological abnormalities in patients with chronic liver disease, especially in patients with liver cirrhosis. It was long believed that the role of platelets in patients with liver cirrhosis was solely to promote aggregation and form the primary haemostatic plug after adhering to the damaged vessel walls through an interaction with von Willebrand factor. (32).

Nowadays, there is growing evidence that platelets, however, play an important role in progression of liver fibrosis through cellular interaction with HSC as well. (10). For example, increasing numbers of platelets as well as platelet-derived chemokine CXCL4 were seen in the immediate vicinity of fibrotic areas in the liver (11). Platelets were shown to stimulate hepatocyte proliferation in vitro through secretion of hepatic - and insulin like growth factor (33). Clinical studies have shown that administration of antithrombotic medication such as aspirin leads to a slowing of the development of fibrosis (34). Interestingly it seems that platelets also may play a role in suppressing HSC activation (35). Therefore, suppressing the activation from a quiescent state to stellate cells producing extracellular matrix proteins that lead to scar formation (36). The regulatory role of platelets in liver fibrosis progression is a subject of ongoing research and the cellular mechanisms are still not fully understood.

The changes in the platelet proteome identified in our study might be associated with an altered platelet response, potentially affecting their role in haemostasis or fibrosis progression. Likely by indirectly influencing pathways affecting the platelet response. Such as the previously mentioned 14-3-3 gamma which interacts with the glycoprotein Ib-IX complex which in turn interplays with VWF or Rho GDI 1 which adjusts Rho GTPase activity and affects platelet physiology and subsequent interactions.

In the past, platelet proteomics were successfully performed in a variety of diseases and in many cases identify molecular and functional platelet changes compared to healthy donors. Yet, a more precise assessment of the consequences of platelet proteome alteration is lacking in many cases.

As with other platelet proteome studies, this analysis is potentially influenced or limited by several factors. Collection and preparation of samples as well as use of non-antithrombotic medications like antidepressants might have influenced platelet activation. Standardization of collection and preparation as well as rigid exclusion criteria were applied to minimize such influences. Due to the numerically very low platelet count in patients with advanced cirrhosis, we were able to extract a limited total amount of platelets from these patients. This limited further evaluation of findings in subsequent analysis such as immunoassays or alternative MS-based methods due to our focus of running each sample both in a technical and biological duplicate. Lastly, a prospective longitudinal approach would have been more conclusive to show changes throughout disease progression.

Page 10/21 
In conclusion, this study is to our knowledge the first to investigate platelet proteome changes in patients with alcohol induced liver cirrhosis. We identified several down-regulated proteins thus providing novel information regarding the platelet proteome and its changes in liver cirrhosis patients. Future studies are needed to further clarify the role and significance of the described proteins due to the many pathways and interactions still unclear in platelet regulation. Additionally future research is now needed to determine the clinical significance for haemostatic or immune regulatory function of platelets in cirrhosis.

\section{Abbreviations}

IPG - Immobilized PH-gradient

TCA - Trichloroacetic acid

IEF - Isoelectric focusing

ACD - Citrate-dextrose solution

INR - international normalized ratio

ESLD - end-stage liver disease

vWF - von Willebrand factor

ACS - Acute Coronary Syndrome

Rab-7a - Ras-related protein 7a

RANBP1 - Ran-specific GTPase activating protein

Rho GDI 1 - Rho-GDP dissociation inhibitor 1

$14-3-3 y-14-3-3$ gamma

HSC - Hepatic stellate cells

RAN - Ras-like nuclear

GPIb-IX - glycoprotein Ib-IX complex

Ras - Rat sarcoma virus

GTP - Guanosine-5'-triphosphate

GDP - guanosine diphosphate

LC-MS - Liquid chromatography-mass spectrometry 
TFA - trifluoroacetic acid

DTT - Dithiothreitol

IAA - lodoacetic acid

SDS - sodium dodecyl sulfate

\section{Declarations}

Ethics approval and consent to participate

The study was previously approved by the ethics committee of the University of Heidelberg and all participants provided written informed consent prior to inclusion in the study.

Consent for publication

Not applicable - no identifiable information

Availability of data and materials

The UniProt database used in this study is available at https://www.uniprot.org/

Competing interests

The authors declare that they have no financial or non-financial competing interests.

Funding

AW received a grant from the Dekanat der Medizinischen Fakultät Heidelberg (Medical faculty Heidelberg). DG was supported by a grant from Deutsche Forschungsgemeinschaft (German Research Foundation). For the publication fee we acknowledge financial support by Deutsche Forschungsgemeinschaft within the funding programme "Open Access Publikationskosten" as well as by Heidelberg University.

Authors' contributions

All authors (Nima Haji Begli, Cora Freund, Karl-Heinz Weiss, Daniel Gotthardt and Andreas Wannhoff) contributed to the study conception and design. Material preparation, data collection and analysis were performed by Nima Haji Begli and Andreas Wannhoff. The first draft of the manuscript was written by Nima Haji Begli and Andreas Wannhoff and Cora Freund, Karl-Heinz Weiss and Daniel Gotthardt commented on previous versions of the manuscript. All authors (Nima Haji Begli, Cora Freund, Karl-Heinz Weiss, Daniel Gotthardt and Andreas Wannhoff) read and approved the final manuscript. 
We thank Petra Kloeters-Plachky (University Hospital Heidelberg) and Yvonne Leopold (University Hospital Heidelberg) for their assistance in processing the samples. Special thanks also go to Renata Blatnik (University of Heidelberg) as well for her help regarding LC-MS measurements and protein identification.

\section{References}

1. Violi F, Basili S, Raparelli V, et al. Patients with liver cirrhosis suffer from primary haemostatic defects? Fact or fiction? J Hepatol. 2011 Dec;55(6):1415-27.

2. Giannini EG, Savarino V. Thrombocytopenia in liver disease. Curr Opin Hematol. 2008 Sep;15(5):47380.

3. Blonski W, Siropaides T, Reddy KR. Coagulopathy in liver disease. Curr Treat Options Gastroenterol. 2007 Dec;10(6):464-73.

4. Tripodi A, Mannucci PM. The coagulopathy of chronic liver disease. N Engl J Med. 2011 Jul $14 ; 365(2): 147-56$.

5. Gulley D, Teal E, Suvannasankha A, et al. Deep vein thrombosis and pulmonary embolism in cirrhosis patients. Dig Dis Sci. 2008 Nov;53(11):3012-7.

6. Tripodi A, Anstee QM, Sogaard KK, et al. Hypercoagulability in cirrhosis: causes and consequences. J Thromb Haemost. 2011 Sep;9(9):1713-23.

7. Semple JW, Italiano JE Jr, Freedman J. Platelets and the immune continuum. Nat Rev Immunol. 2011 Apr;11(4):264-74.

8. Garraud O, Chabert A, Hamzeh-Cognasse $\mathrm{H}$, et al. Platelets and immunity: From physiology to pathology. Transfus Clin Biol. 2017 Jun;24(2):83-86.

9. Kisucka J, Butterfield CE, Duda DG, et al. Platelets and platelet adhesion support angiogenesis while preventing excessive hemorrhage. Proc Natl Acad Sci U S A. 2006 Jan 24;103(4):855-60.

10. Sullivan BP, Wang R, Tawfik O, et al. Protective and damaging effects of platelets in acute cholestatic liver injury revealed by depletion and inhibition strategies. Toxicol Sci. 2010 May;115(1):286-94.

11. Zaldivar MM, Pauels K, von Hundelshausen P, et al. CXC chemokine ligand 4 (Cxcl4) is a plateletderived mediator of experimental liver fibrosis. Hepatology. 2010 Apr;51(4):1345-53.

12. Mateos-Cáceres PJ, Macaya C, Azcona L, et al. Different expression of proteins in platelets from aspirin-resistant and aspirin-sensitive patients. Thromb Haemost. 2010 Jan;103(1):160-70.

13. Arias-Salgado EG, Larrucea S, Butta N, et al. Variations in platelet protein associated with arterial thrombosis. Thromb Res. 2008;122(5):640-7.

14. Fecher-Trost C, Wissenbach U, Beck A, et al. The in vivo TRPV6 protein starts at a non-AUG triplet, decoded as methionine, upstream of canonical initiation at AUG. J Biol Chem. 2013 Jun 7;288(23):16629-16644.

15. Keller A, Nesvizhskii Al, Kolker E, et al. Empirical statistical model to estimate the accuracy of peptide identifications made by MS/MS and database search. Anal Chem. 2002 Oct 15;74(20):5383-92. 
16. Nesvizhskii Al, Keller A, Kolker E, et al. A statistical model for identifying proteins by tandem mass spectrometry. Anal Chem. 2003 Sep 1;75(17):4646-58.

17. Cantalupo G, Alifano P, Roberti V, et al. Rab-interacting lysosomal protein (RILP): the Rab7 effector required for transport to lysosomes. EMBO J. 2001;20(4):683-693.

18. Walsh TG, Li Y, Wersäll A, et al. Small GTPases in platelet membrane trafficking. Platelets. 2019;30(1):31-40.

19. Rendu F, Brohard-Bohn B. The platelet release reaction: granules' constituents, secretion and functions. Platelets. 2001;12(5):261-273.

20. Guerrier L, Claverol S, Fortis F, et al. Exploring the platelet proteome via combinatorial, hexapeptide ligand libraries. J Proteome Res. 2007 Nov;6(11):4290-303.

21. Ren M, Villamarin A, Shih A, et al. Separate domains of the Ran GTPase interact with different factors to regulate nuclear protein import and RNA processing. Mol Cell Biol. 1995 Apr;15(4):2117-24.

22. Burkhart JM, Vaudel $M$, Gambaryan $S$, et al. The first comprehensive and quantitative analysis of human platelet protein composition allows the comparative analysis of structural and functional pathways. Blood. 2012 Oct 11;120(15):e73-82.

23. Garcia-Mata R, Boulter E, Burridge K. The 'invisible hand': regulation of RHO GTPases by RHOGDIs. Nat Rev Mol Cell Biol. 2011 Jul 22;12(8):493-504.

24. Goggs R, Williams CM, Mellor H, et al. Platelet Rho GTPases-a focus on novel players, roles and relationships. Biochem J. 2015 Mar 15;466(3):431-42.

25. Li W, Wang H, Jin X, Zhao L, et al. Loss of RhoGDI is a novel independent prognostic factor in hepatocellular carcinoma. Int J Clin Exp Pathol. 2013 2013;6(11):2535-2541

26. Wheeler-Jones $\mathrm{CP}$, Learmonth MP, Martin H, et al. Identification of 14-3-3 proteins in human platelets: effects of synthetic peptides on protein kinase C activation. Biochem J. 1996 Apr 1;315 ( Pt 1)(Pt 1):41-7.

27. Gegenbauer K, Nagy Z, Smolenski A. Cyclic nucleotide dependent dephosphorylation of regulator of G-protein signaling 18 in human platelets. PLoS One. 2013 Nov 7;8(11):e80251.

28. Du X. Signaling and regulation of the platelet glycoprotein Ib-IX-V complex. Curr Opin Hematol. 2007 May;14(3):262-9.

29. Cosemans JM, Schols SE, Stefanini L, et al. Key role of glycoprotein Ib/V/IX and von Willebrand factor in platelet activation-dependent fibrin formation at low shear flow. Blood. 2011;117(2):651-660

30. Chen Y, Ruggeri ZM, Du X. 14-3-3 proteins in platelet biology and glycoprotein Ib-IX signaling. Blood. 2018;131(22):2436-2448.

31. Estevez B, Kim K, Delaney MK, et al. Signaling-mediated cooperativity between glycoprotein Ib-IX and protease-activated receptors in thrombin-induced platelet activation. Blood. 2016 Feb 4;127(5):62636.

32. Hugenholtz GGC, Porte RJ, Lisman T. The platelet and platelet function testing in liver disease. Clin Liver Dis. 2009 Feb;13(1):11-20. 
33. Matsuo R, Ohkohchi N, Murata S, et al. Platelets Strongly Induce Hepatocyte Proliferation with IGF-1 and HGF In Vitro. J Surg Res. 2008 Apr;145(2):279-86.

34. Simon TG, Henson J, Osganian S, et al. Daily Aspirin Use Associated With Reduced Risk For Fibrosis Progression In Patients With Nonalcoholic Fatty Liver Disease. Clin Gastroenterol Hepatol. 2019 Dec;17(13):2776-2784.e4.

35. Kodama $\mathrm{T}$, Takehara $\mathrm{T}$, Hikita $\mathrm{H}$, et al. Thrombocytopenia exacerbates cholestasis-induced liver fibrosis in mice. Gastroenterology. 2010 Jun;138(7):2487-98, 2498.e1-7.

36. Friedman SL. Hepatic stellate cells: protean, multifunctional, and enigmatic cells of the liver. Physiol Rev. 2008;88(1):125-172.

\section{Supplementary Figures}

Supplementary Figures are not available with this version.

\section{Figures}



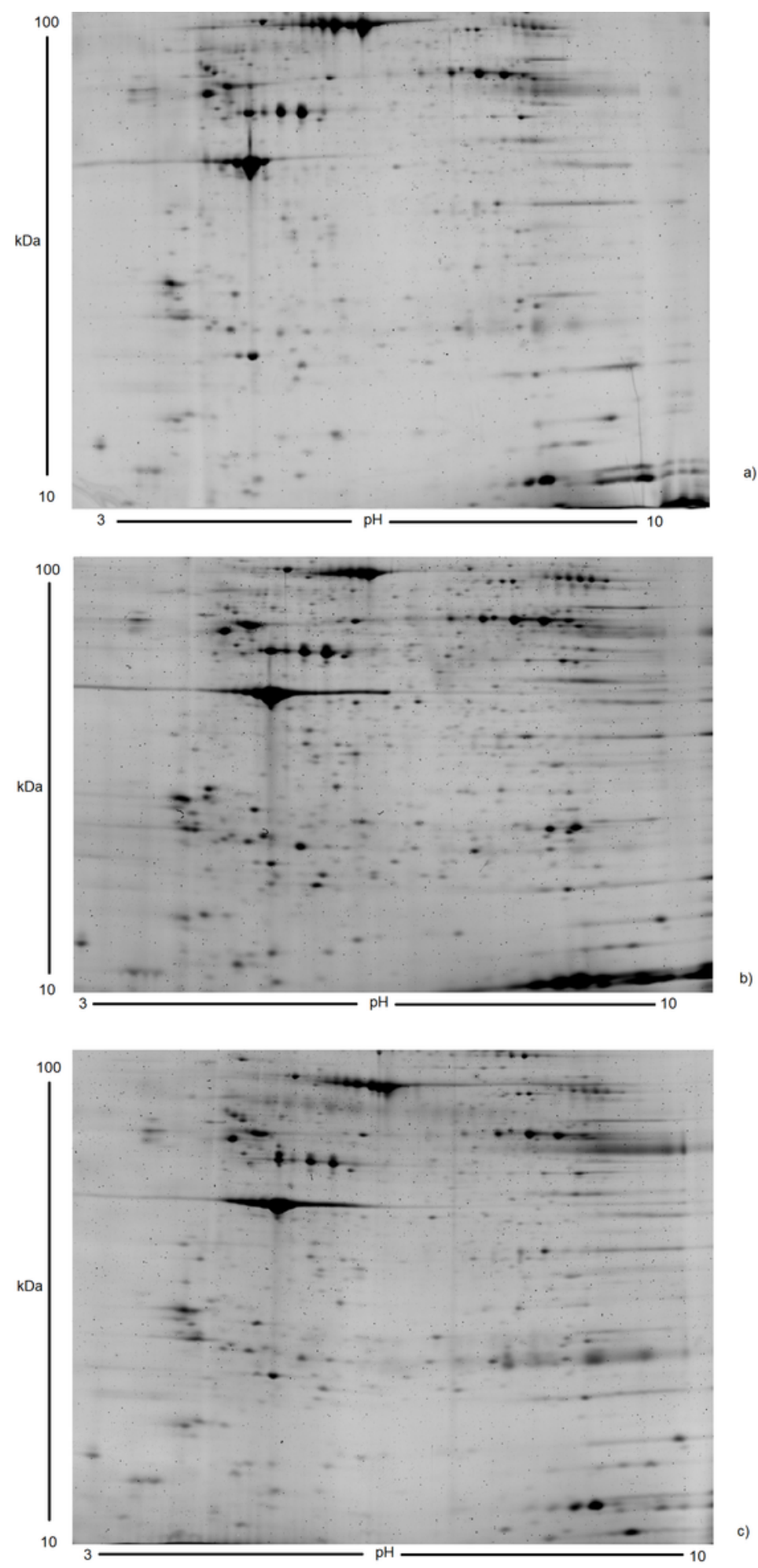

\section{Figure 1}

\section{Two-dimensional reference gels}

Two-dimensional reference gels of six healthy controls (a), six patients with less advanced alcohol induced cirrhosis (b) and advanced disease respectively (c). Each sample was run both in a technical and 
biological duplicate. Reference gels were obtained using the proprietary parameters preset in the analysis program. Original gels are presented in Supplementary Figure 1-3.

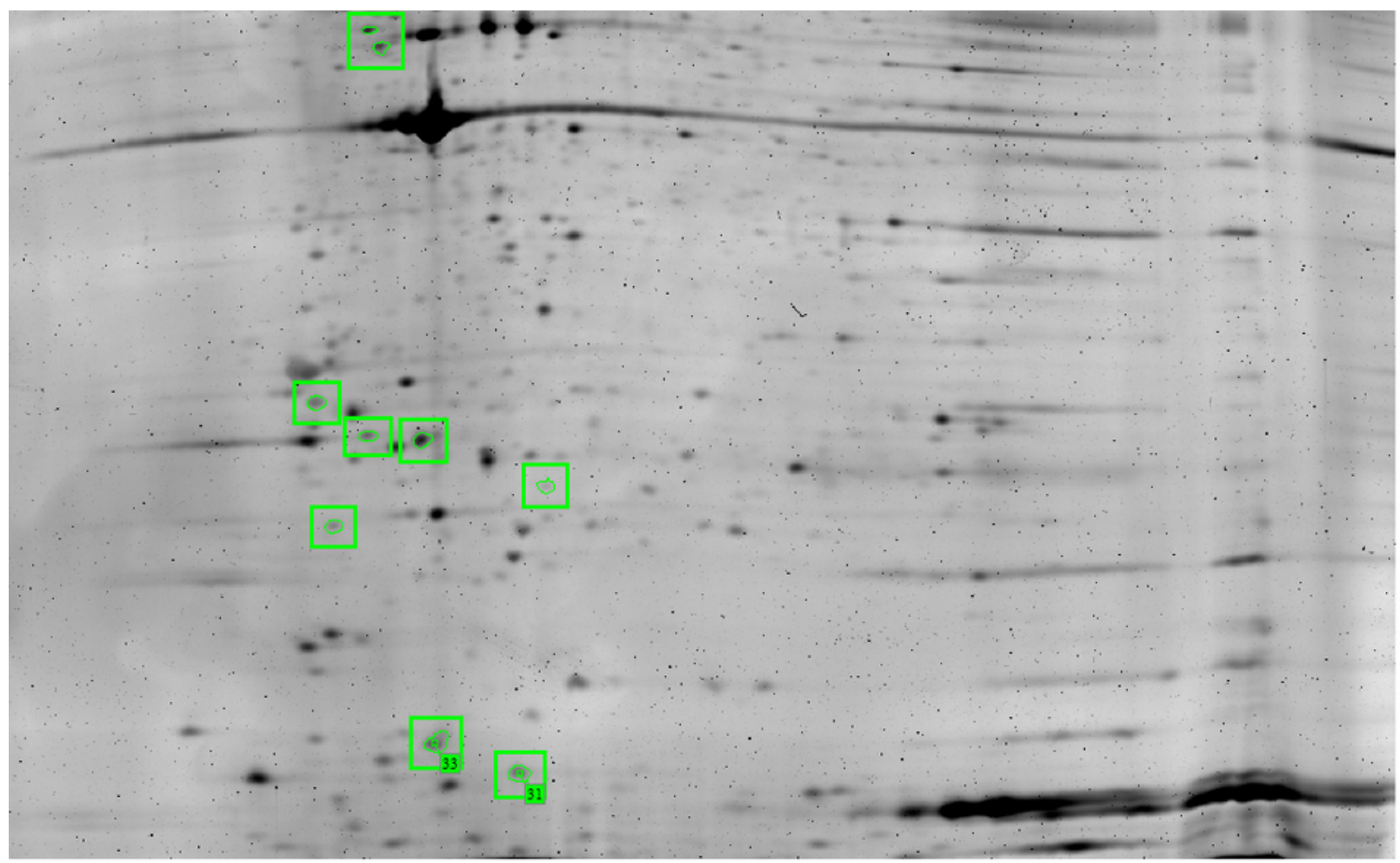

a)

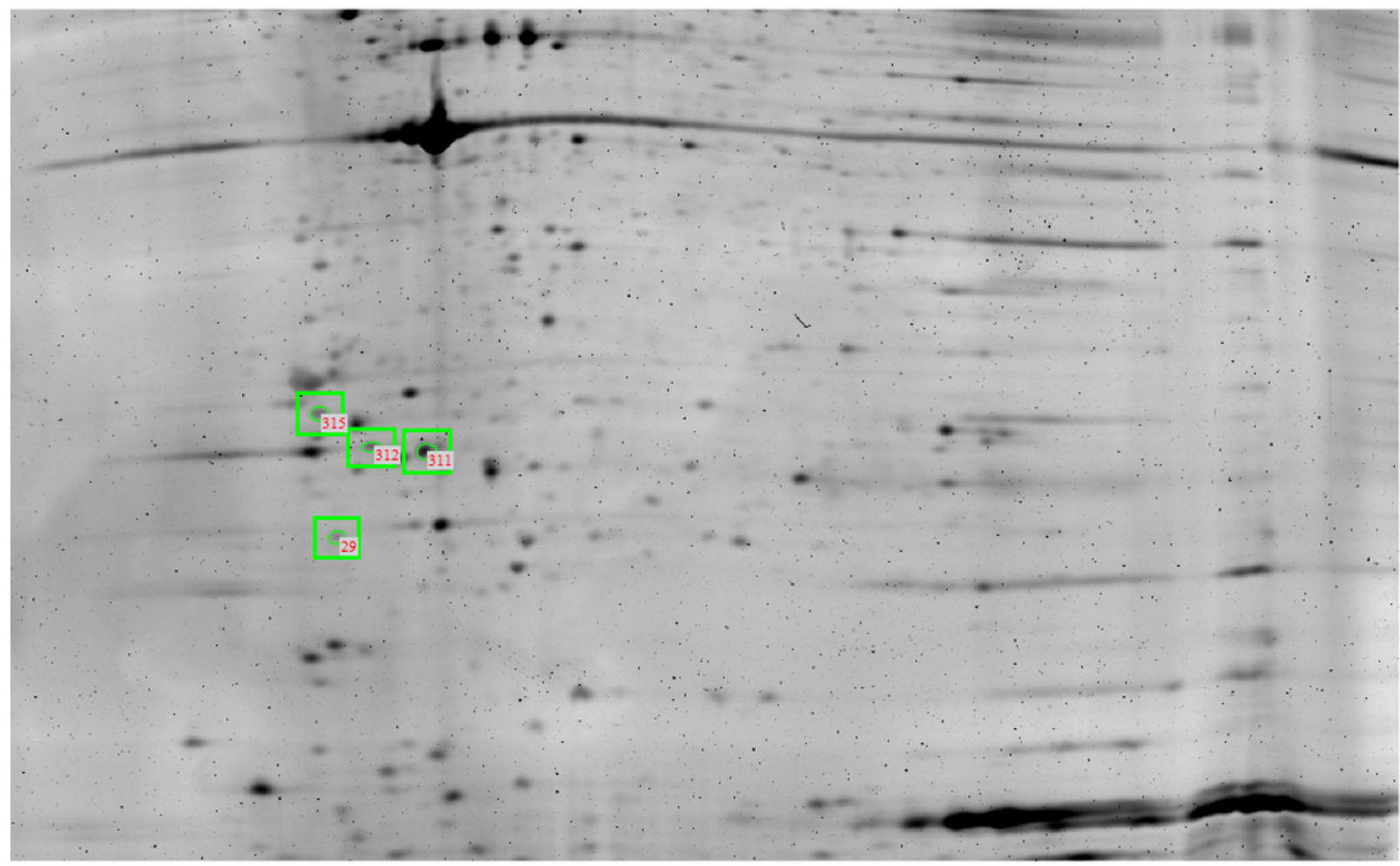

b)

Figure 2

Statistically significant protein spots 
Nine spots (a) (highlighted in green) in total fulfilled the criteria to be accepted as statistically significant (2-fold abundance change and an ANOVA $<0.05$ ) and were selected for protein identification. Four of them (b) were spots without mixed protein profiles and showed platelet proteins. Original gel is presented in Supplementary Figure 4.

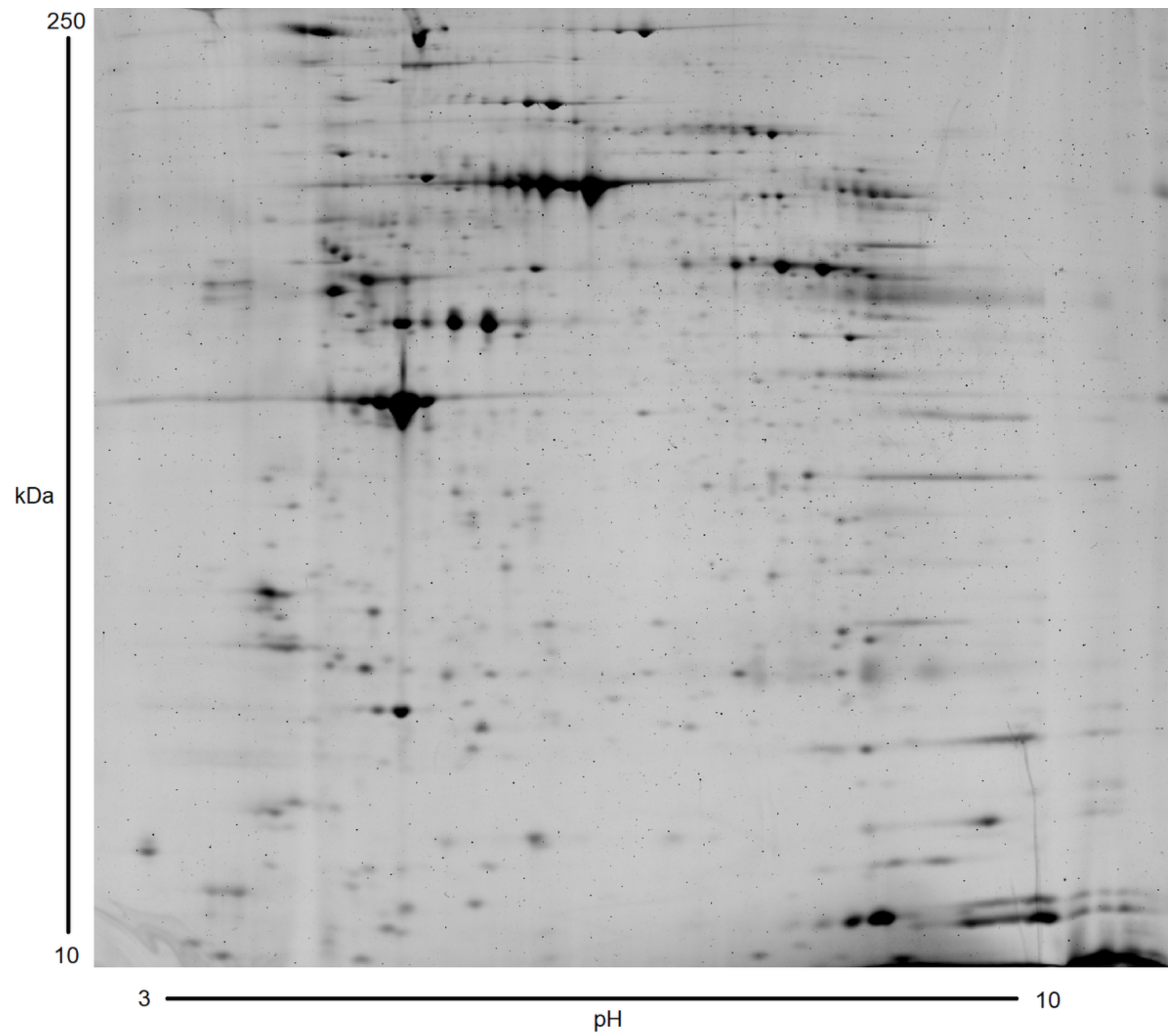

Figure 3

\section{D gel of healthy control group}

Exemplary two-dimensional gel of a healthy control. Original gel is presented in Supplementary Figure 1. 


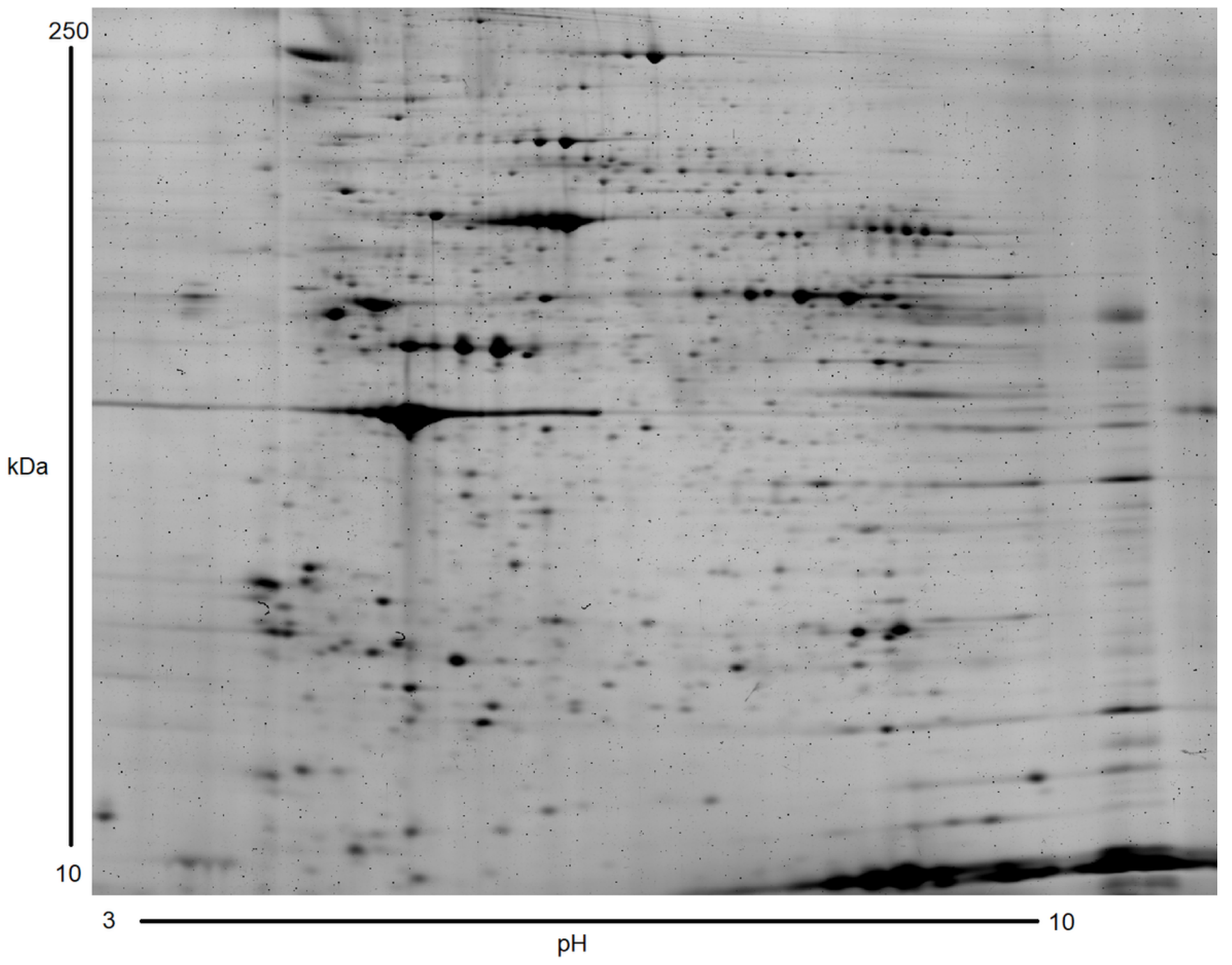

Figure 4

2D-gel of less advanced cirrhosis group

Exemplary two-dimensional gel of a patient with less advanced alcohol induced cirrhosis. Original gel is presented in Supplementary Figure 2. 


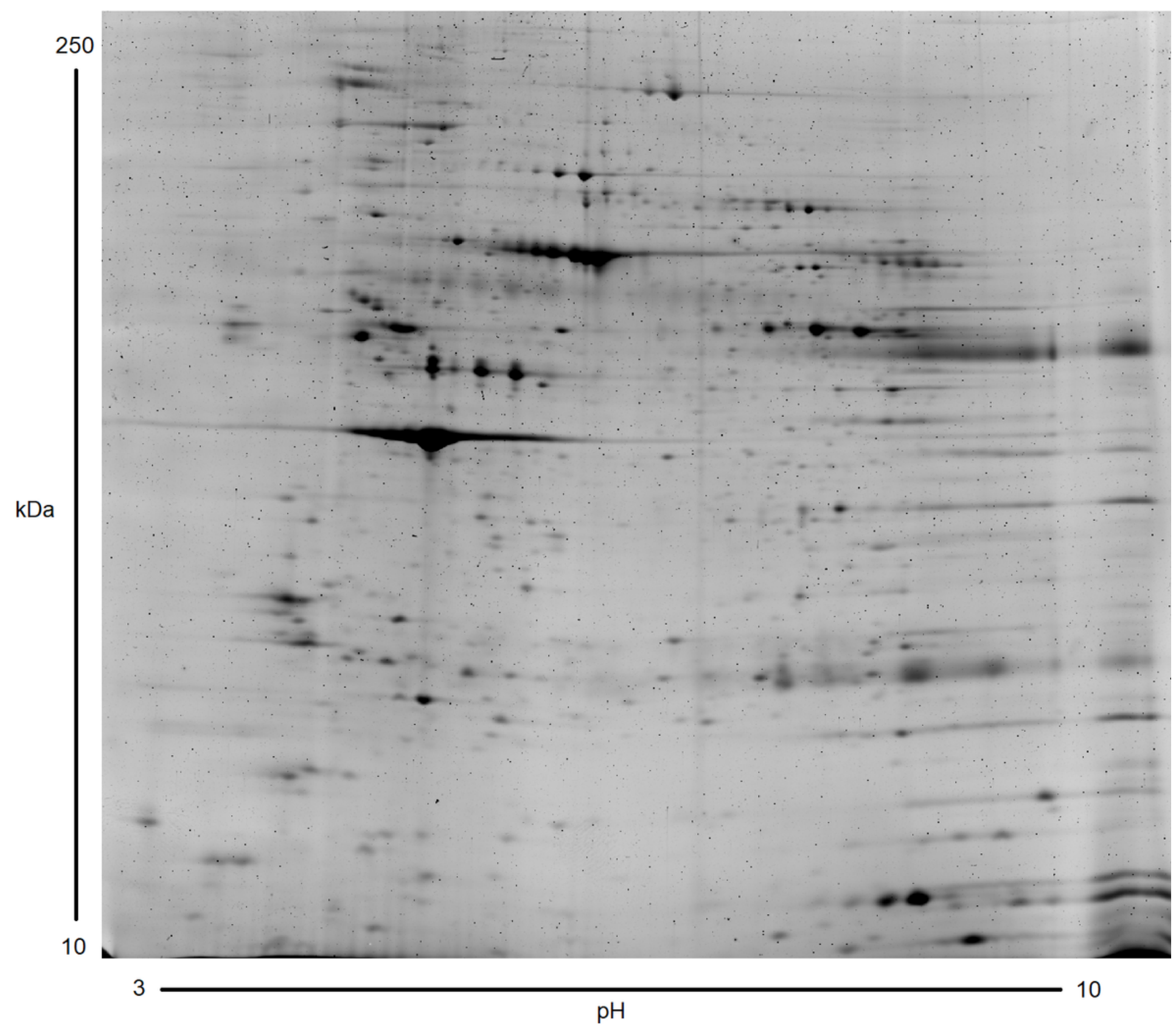

Figure 5

2D-gel of advanced cirrhosis group

Exemplary two-dimensional gel of a patient with advanced alcohol induced cirrhosis. Original gel is presented in Supplementary Figure 3. 


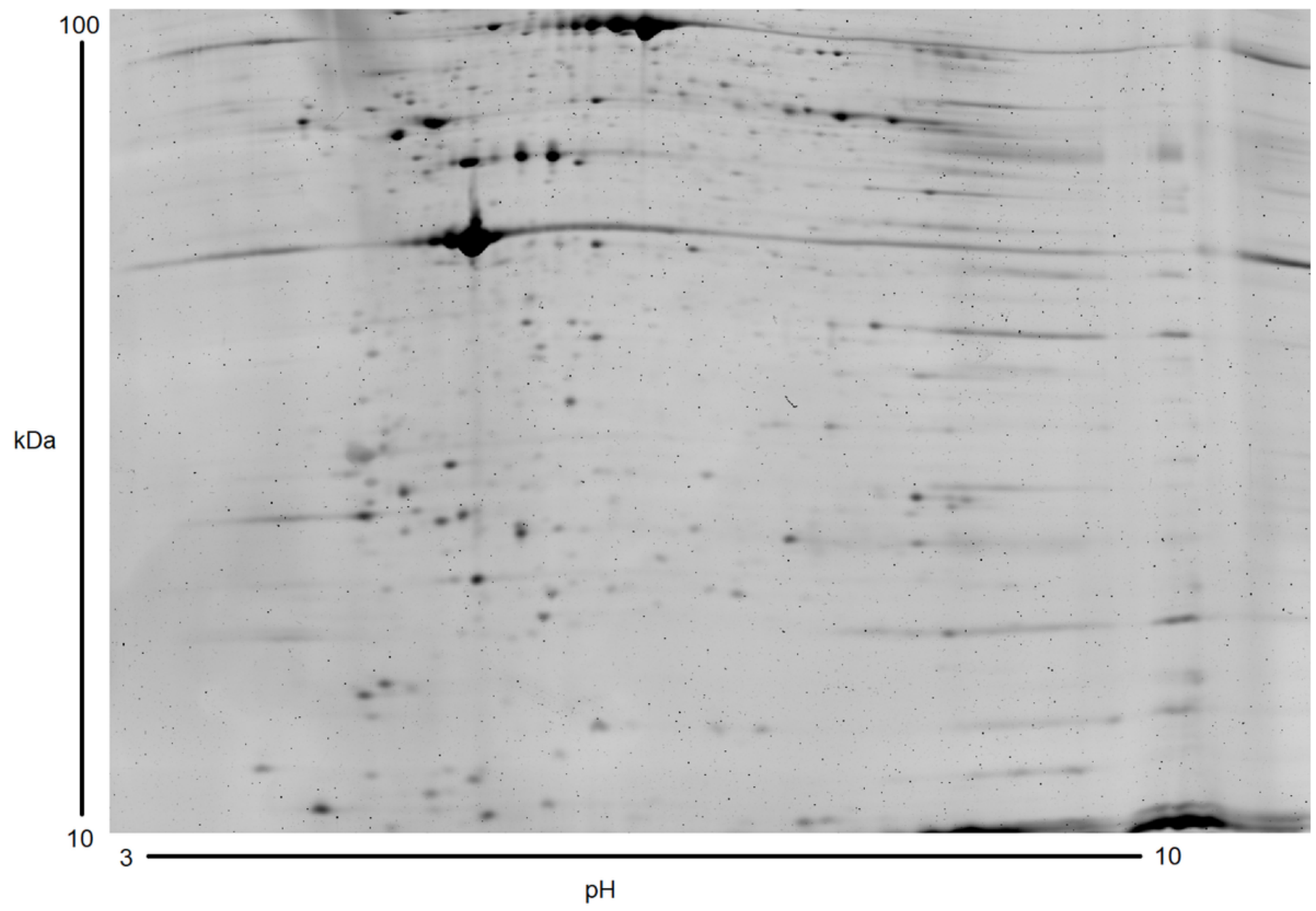

Figure 6

Exemplary two-dimensional gel of a healthy patient

Exemplary two-dimensional gel of a healthy patient used in fig. 2 to illustrate the statistically significant proteins. Original gel is presented in Supplementary Figure 4.

\section{Supplementary Files}

This is a list of supplementary files associated with this preprint. Click to download.

- Figure3SourceRev300.tif

- Figure4SourceRev300.tif

- Figure5SourceRev300.tif

- Figure6SourceRev300.tif 